This item was submitted to Loughborough's Research Repository by the author.

Items in Figshare are protected by copyright, with all rights reserved, unless otherwise indicated.

Accuracy of harmonic analysis techniques for direction of arrival estimation using time modulated arrays

PLEASE CITE THE PUBLISHED VERSION

https://doi.org/10.1049/cp.2018.1032

PUBLISHER

IEEE

VERSION

AM (Accepted Manuscript)

LICENCE

CC BY-NC-ND 4.0

REPOSITORY RECORD

Clark, Ben, and James A. Flint. 2019. "Accuracy of Harmonic Analysis Techniques for Direction of Arrival Estimation Using Time Modulated Arrays”. figshare. https://hdl.handle.net/2134/33394. 


\title{
Accuracy of Harmonic Analysis Techniques for Direction of Arrival Estimation Using Time Modulated Arrays
}

\author{
Ben Clark ${ }^{1}$, James A. Flint ${ }^{1}$ \\ ${ }^{1}$ Wolfson School of Mechanical, Electrical, and Manufacturing Engineering, \\ Loughborough University, Loughborough, Leicestershire, LE11 3TU, BClark@theiet.org
}

\begin{abstract}
This paper presents a comparison between two direction of arrival (DoA) estimation methods using time modulated linear arrays (TMLAs). The first method uses a two element array and numerically derives the signal DoA by using the ratio of the two harmonics created by array. The second method makes use of a greater number of elements and known steering angles of the array sidebands. Results of a numerical simulation are presented which detail the accuracy in direction finding when using the two techniques while the array is subject to environmental conditions such as noise and coherent signal interference. It is shown that whilst the second method requires a greater number of elements, it has greater immunity to noise and multipaths.
\end{abstract}

Index Terms - time modulation, array, direction of arrival.

\section{INTRODUCTION}

Time Modulated Arrays (TMAs) were introduced by Shanks and Bickmore [1], and in 1961, Shanks showed how these arrays could be used to form simultaneous beams which operate at different frequencies [2]. In summary, time modulation is realised by switching individual elements in an array on and off periodically. The periodic nature of the switching causes an incident signal to have its power distributed into a number of sidebands that exist at unique frequencies and depend on the angle of incidence and the switching pattern.

TMAs offer reduced complexity over conventional arrays as they do not require a phase shift network in order to produce a beam pattern. The switching circuit is controlled digitally, and therefore a wide range of beam patterns can arise out of the possible combinations without having to alter the basic setup of the array [3], [4].

Much of the literature on TMAs focuses on reducing the sidebands created by the array [5], [6], [7]; however, recent research has been made into using the sidebands for use in Direction of Arrival (DoA) estimation. Tennant and Chambers [8] showed that by adjusting the on-time ratio of a two element array, it was possible to steer a deep null region which could be used for target scanning. This technique requires the null to be steered across a range of directions before an estimation can be derived.

He et al. [9] showed that the harmonics of a two element array could be used to find the target without sweeping a particular beam. It was shown that a DoA estimation can be obtained using the ratio of the frequency components produced by the array. Using this method, only one set of data-points need to be captured to find a signal within a $\pm 90^{\circ}$ range.

In ideal conditions, He et al.'s solution has zero error, and can estimate the DoA of multiple incoherent signals simultaneously. However, there is limited information about how effective the algorithm is in a practical situation where there is noise and multiple coherent signals, that originate from multipaths for example. An alternative DoA finding method was proposed that makes use of a larger number of sensors [10] and compares the real-valued powers of the two largest adjacent harmonic components and uses their known mainbeam angles to linearly interpolate a DoA estimation. This method was demonstrated in the acoustics domain, but is also applicable to antenna arrays.

The present paper compares the effectiveness of the two harmonic analysis techniques when subject to realistic conditions. First, the paper simulates a scenario where a target is to be located in the presence of white, Gaussian-distributed noise. Then, the two methods are used to locate a target in the presence of an interfering coherent signal, such as one that might originate from a multipath.

\section{BACKGROUND THEORY}

The output $y(t)$ of an array at time $t$ for a time modulated linear array (TMLA) consisting of $N$ elements and being illuminated by a far-field signal $x(t)$ as shown in Fig. 1 is given as [5]:

$$
y(t)=\sum_{n=0}^{N-1} x(t) U_{n}(t) e^{j k n d \sin \theta}
$$

where $k$ is the wave number, $d$ is the element spacing and $\theta$ is the incident angle relative to the broadside of the array. $U_{n}(t)$ is a periodic switching function that controls the element amplitude at any time. In the case of a simple TMA, this switching function is either 1 or 0 to represent an element in the array being switched 'on' or 'off' at a particular point in time.

Since $U_{n}(t)$ is periodic with time $T_{s}$, then the array factor can be represented in the frequency domain as a Fourier series. Frequencies of an input signal are distributed across a series of harmonics which are separated by frequency $f_{s}=1 / T_{s}$ and are centred on an input frequency $f_{0}$. Due to 


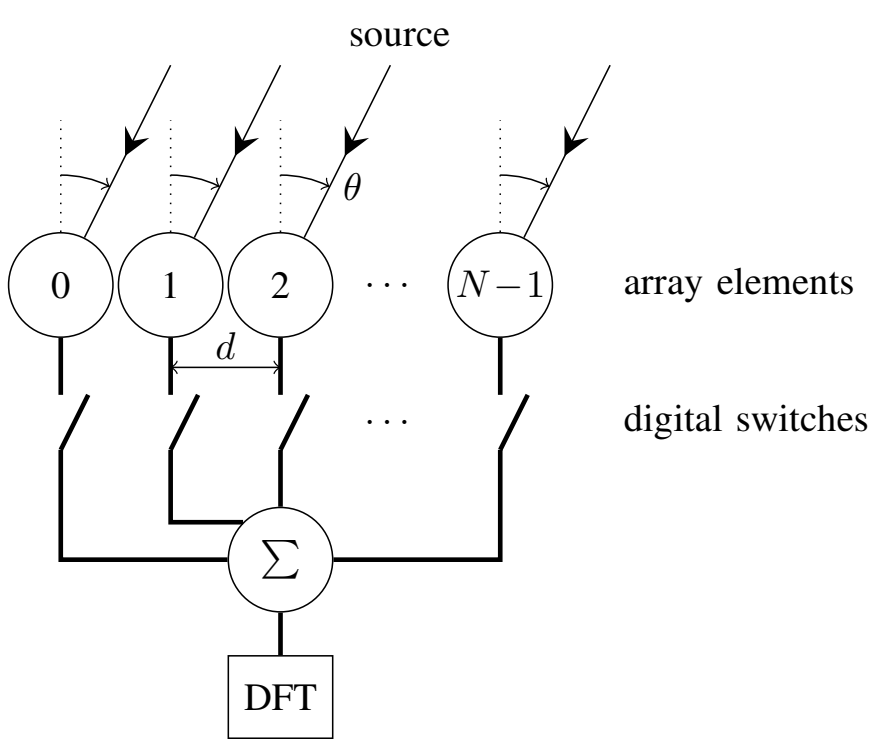

Fig. 1. A typical setup of a Time Modulated Linear Array (TMLA) consisting of $N$ elements. In the present paper, only one element is switched on at any time.

the periodic changes of signal phase as the array is switched from one element to another, each of these harmonics have an independent response to different signal DoAs [9], [10]. The complex amplitude for a particular harmonic $h$ can be calculated by performing a Discrete Fourier Transform (DFT) at the frequency $f_{0}+h f_{s}$.

\section{A. Harmonic Characteristic (HC) Method}

He et al.'s method uses the real and imaginary parts of the FFT taken from the output of a two element TMLA switched sequentially. A DoA estimation can be derived using the centre and outer harmonics $X_{0}$ and $X_{1}$ respectively [9]:

$$
\theta_{\text {est }}=\arcsin \left(\frac{2}{k d} \arctan \left(\frac{\pi X_{1}}{2 X_{0}}\right)\right)
$$

Equation (2) is derived analytically, so the only source of error is numerical error. The method can locate a signal within a $\pm 90^{\circ}$ angular range, however there is an assumption of ideal conditions, i.e. no interference.

\section{B. Weighted Average (WA) Method}

An alternative method uses the power values in the harmonic bins of an array. For example, an array with five elements will have five distinct responses at the frequencies $f_{0} \pm 2 f_{s}, f_{0} \pm 1 f_{s}$ and $f_{0}$ as shown in Fig. 2. A TMLA of $N$ elements will have harmonics indexed in the range of $\pm N / 2$ and each of these sidebands have a main beam which have direction

$$
\theta_{h}=\arcsin \left(\frac{h}{R N}\right)
$$

where $R$ is the ratio between signal wavelength and element distance. At the angular point at which each of these sidebands have a maximum response, all other sidebands have a minimal

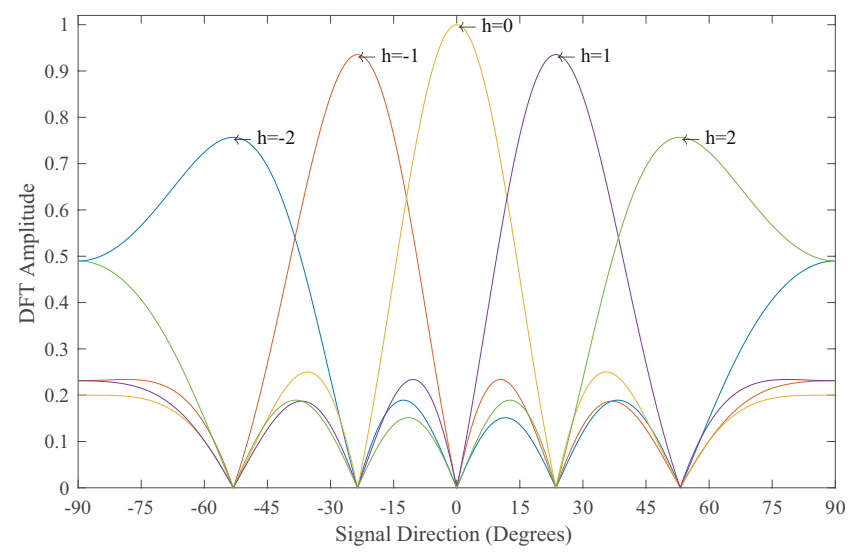

Fig. 2. The array factor for a 5-element array, showing the frequency response of five different sidebands indexed by $h$.

response. This comparative relationship can be exploited to linearly derive a DoA estimation using the two largest adjacent harmonics $X_{\alpha}$ and $X_{\alpha+1}$ and their known main beam angles $\theta_{\alpha}$ and $\theta_{\alpha+1}$ respectively [10]:

$$
\theta_{\text {est }}=\frac{\left(X_{\alpha} \theta_{\alpha}\right)+\left(X_{\alpha+1} \theta_{\alpha+1}\right)}{X_{\alpha}+X_{\alpha+1}}
$$

Unlike He et al.'s method, there are theoretical limitations inherent from this method as it involves interpolation. However, the purpose of the paper is to demonstrate that the interpolation has some benefits in improving noise immunity compared to the original method. It can be shown that the theoretical limitations are less significant as the number of elements in the array increases, even when keeping the total number of captured samples the same. It should also be noted that only one input stage is required for data acquisition regardless of the number of elements used, since only one element is expected to be on at any one time for the purposes of DoA estimation.

\section{NumericAl Simulation}

To compare the two methods, a numerical simulation was used. A $1 \mathrm{GHz}$, single-tone sinusoidal signal was assumed, and modelled as impinging at an angle $\theta$, on a linear array with isotropic elements spaced half a wavelength apart. For each angle measured within a $\pm 90^{\circ}$ range, 1350 samples were generated at a rate of 10 Gsamples/sec. During the generation process the effect of time modulation was implemented by introducing periodic delays related to the element position. For the harmonic characteristic method, two elements were used; for the weighted average method, five, nine and fifteen elements were used. Since the total number of samples remained constant throughout each simulation, this meant that for a 2-element array 675 samples were taken for each element consecutively; for a 5-element array, 270 samples were taken for each element consecutively etc.

Fig. 3 shows the typical estimation error for each method without interference. It appears that the harmonic characteristic method is the preferred method for DoA estimations in this 


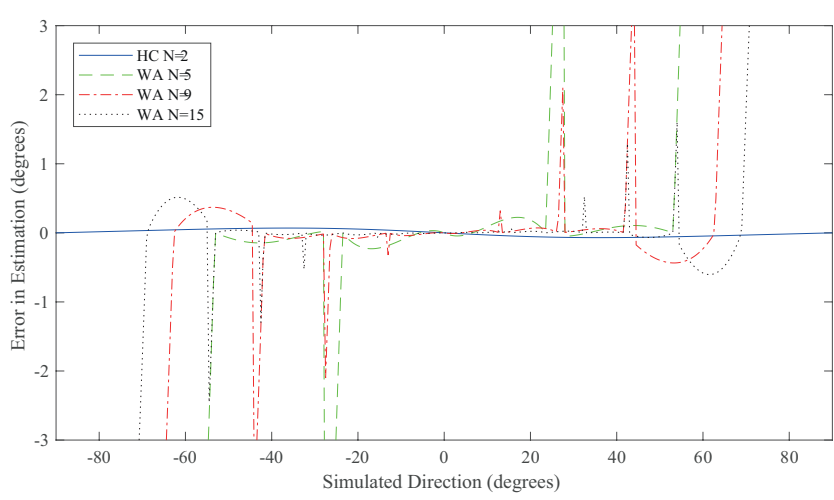

Fig. 3. Simulated error in direction finding when using the harmonic characteristic method (HC) and the weighted average method (WA) for 5 , 9 and 15 elements, and ideal environmental conditions.

scenario since the errors are entirely numerical, and the angular range of accurate estimation is greater than that of the weighted average method.

The mean computation time of the 100 simulations was taken. This was measured from the time that the DFTs are complete to the time at which a DoA estimation is obtained). In The weighted average method, this included the time in finding the largest DFT and the level of its adjacent sideband and took $0.87 \mu \mathrm{s}, 0.96 \mu \mathrm{s}$ and $1.14 \mu \mathrm{s}$ to estimate the DoAs using 5, 9 and 15 elements respectively. For the harmonic characteristic method, the elapsed time was $1.31 \mu \mathrm{s}$, showing that the weighted average method is computationally simpler, although it should be noted that a greater number of DFTs are required when using the weighted average method with a greater number of elements and this cannot always be done in parallel to sampling.

In the following sections, common environmental situations are considered. For each variable changed, 100 simulations were carried out and the best-case and worst-case (as measured by the root mean squared error (RMSE) between $\pm 80^{\circ}$ ) results were recorded. The starting phase in each simulation was changed to a random position, since the phase is unknown in the real world.

\section{A. Estimations in the Presence of Noise}

The simulation was modified to include Gaussian distributed noise. The output of the array in the presence of noise $n(t)$ is assumed to be:

$$
y(t)=n(t)+\sum_{n=0}^{N-1} x(t) U_{n}(t) e^{j k n d \sin \theta}
$$

Results are produced based on varying levels of noise. For each repeated test, DoA results were fairly similar, so only the worst case simulation is shown. Fig. 4, Fig. 5 and Fig. 6 show the error in direction finding in the presence of noise at $20 \mathrm{~dB}$, $10 \mathrm{~dB}$ and $0 \mathrm{~dB}$ signal-to-noise ratio (SNR) respectively. It can be seen that the weighted average method is generally more noise immune, however the harmonic characteristic method

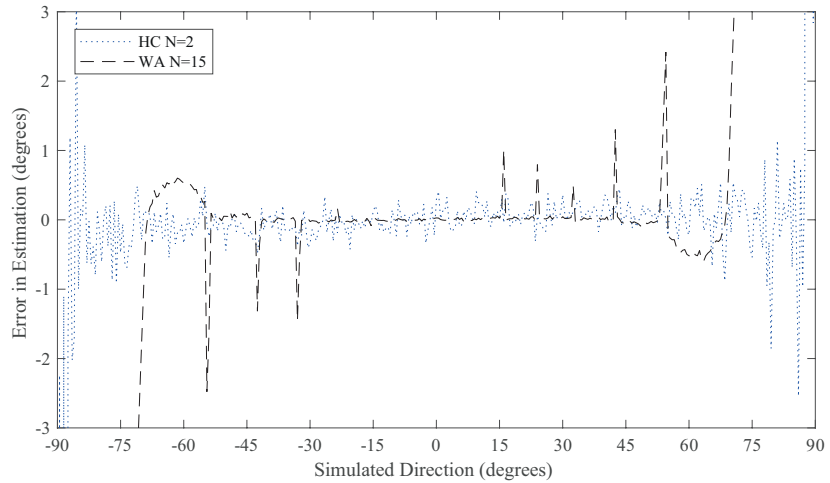

Fig. 4. Worst case simulated error in direction finding when using the harmonic characteristic method (HC) using 2 elements and the weighted average method (WA) for 15 elements, when the array is illuminated in the presence of noise at $20 \mathrm{~dB}$ SNR.

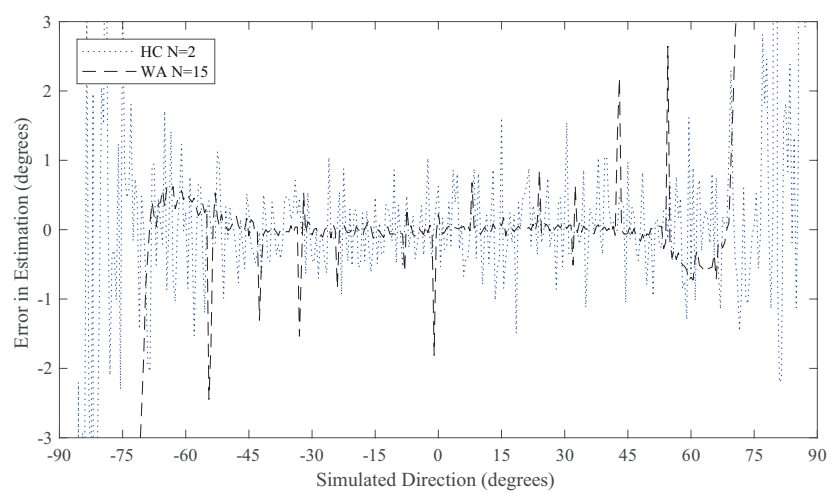

Fig. 5. Worst case simulated error in direction finding when using the harmonic characteristic method (HC) using 2 elements and the weighted average method (WA) for 15 elements, when the array is illuminated in the presence of noise at $10 \mathrm{~dB}$ SNR.

maintains a greater angular range. It can also be seen that the error in estimation using the weighted average method is greatest at the angles calculated by (3). Errors at these specific regions when using the weighted average method become similar to the errors at any position using the harmonic characteristic method at noise levels greater than $10 \mathrm{~dB}$ SNR.

With decreased SNR, the difference in noise immunity becomes apparent. It should be noted that this is the case for a small number of samples taken. The harmonic characteristic method has been shown to perform well with larger sample counts in [9].

\section{B. Estimations in the Presence of an Interfering Signal}

The simulation was modified further to include a secondary interfering signal. The interfering signal was set at the same frequency but at $10 \%$ amplitude compared to the target signal and was kept at a fixed angle of $30^{\circ}$. In this scenario, the best and worst results are given in Fig. 7 and Fig. 8 respectively and the phases of the source and interfering signals in those cases are given as $\phi_{s}$ and $\phi_{i}$ respectively. 


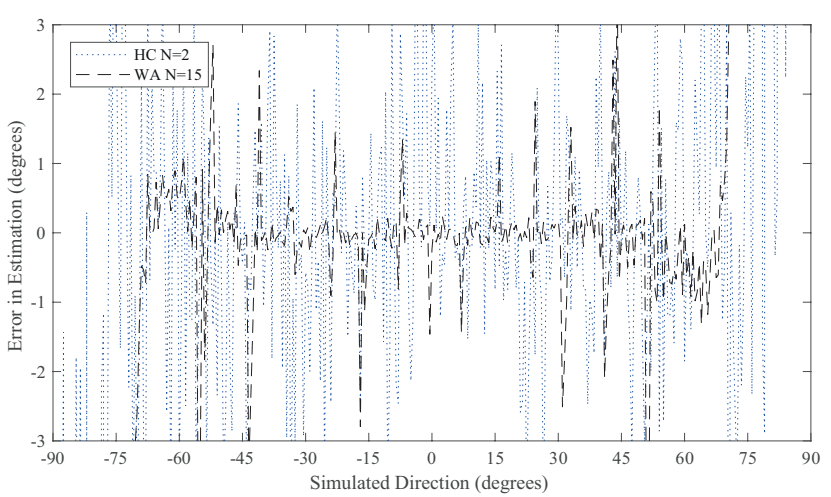

Fig. 6. Worst case simulated error in direction finding when using the harmonic characteristic method (HC) using 2 elements and the weighted average method (WA) for 15 elements, when the array is illuminated in the presence of noise at $0 \mathrm{~dB}$ SNR.

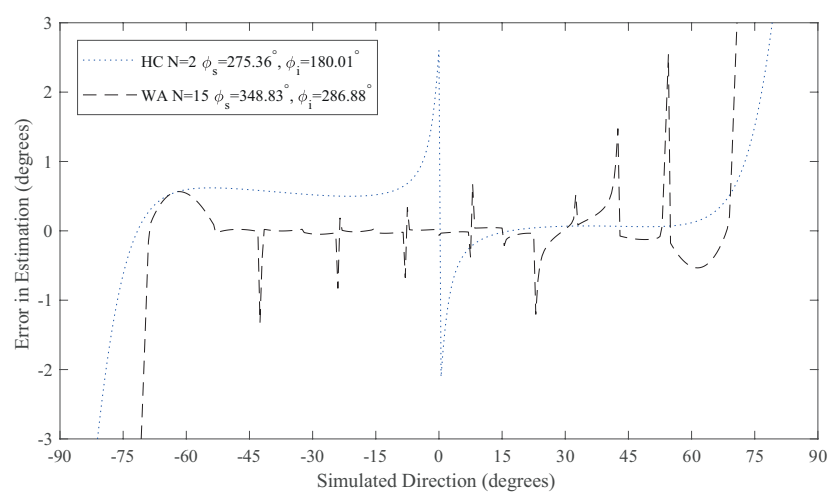

Fig. 7. Best case simulated error in direction finding when using the harmonic characteristic method (HC) using 2 elements and the weighted average method (WA) for 15 elements, when the array is illuminated with an interference signal at $30^{\circ}$.

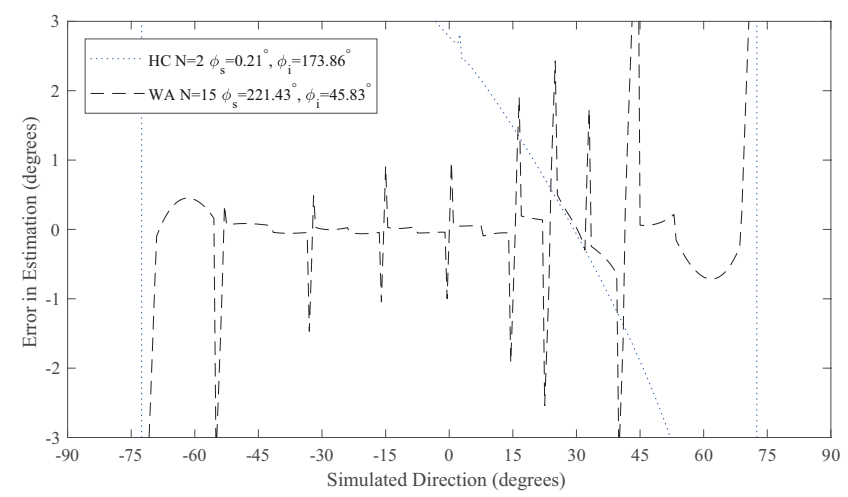

Fig. 8. Worst case simulated error in direction finding when using the harmonic characteristic method (HC) using 2 elements and the weighted average method (WA) for 15 elements, when the array is illuminated with an interference signal at $30^{\circ}$.

As can be seen in the figures, the weighted average method maintains a similar result to its measurement during ideal conditions, but is most affected near the region of the interferer. The results do not vary significantly between best and worst case which suggests a good immunity to multiple coherent signals. Conversely, the harmonic characteristic analysis method varies greatly between best-case and worst-case simulations. It is clear that the accuracy of this method depends greatly on the phase of the incoming signals.

\section{CONCLUSION}

The harmonic characteristic method should be the preferred choice when determining the DoA in ideal conditions. However in the presence of noise or interference from a coherent signal, the weighted average method offers a good alternative whilst maintaining the small computational effort. Methods of reducing the spikes of inaccuracies in the weighted average method have been discussed in [10]; however, the caveat of using this method is the use of a greater number of elements in the array which increases the cost of the system, especially since the number of switches needed will also increase. The number of operations required to obtain a DoA estimation also increases as a DFT needs to be performed at a greater number of frequencies; however, the use of time-modulation means that only one input stage is necessary regardless of the number of elements used.

\section{ACKNOWLEDGMENT}

The authors are grateful for the financial support of Loughborough University's mini Centre for Doctorial Training (mCDT) in "Building a research future in digital communications".

\section{REFERENCES}

[1] H. E. Shanks and R. W. Bickmore, "Four-Dimensional Electromagnetic Radiators," Can. J. Phys., vol. 37, no. 3, pp. 263-275, mar 1959.

[2] H. E. Shanks, "A new technique for electronic scanning," IRE Trans. Antennas Propag., vol. 9, no. 2, pp. 162-166, mar 1961.

[3] M. M. Abusitta, R. A. Abd-Alhameed, I. T. E. Elfergani, A. D. Adebola, and P. S. Excell, "Beam Steering of Time Modulated Antenna Arrays Using Particle Swarm Optimization," in PIERS Proceedings. Marrakesh: PIERS, 2011, pp. 488-452.

[4] Y. Tong and A. Tennant, "Simultaneous control of sidelobe level and harmonic beam steering in time-modulated linear arrays," Electron. Lett., vol. 46, no. 3, p. 200, 2010.

[5] L. Poli, P. Rocca, L. Manica, and A. Massa, "Handling Sideband Radiations in Time-Modulated Arrays Through Particle Swarm Optimization," IEEE Transactions on Antennas and Propagation, vol. 58, no. 4, pp. 1408-1411, apr 2010.

[6] E. Afacan, "Sidelobe Level and Sideband Optimization for Thinned Planar Antenna Arrays Using Time Modulation," in 2013 7th European Conference on Antennas and Propagation (EuCAP), no. Eucap. IEEE, 2013, pp. 328-330.

[7] C. He, H. Yu, X. Liang, J. Geng, and R. Jin, "Sideband Radiation Level Suppression in Time-Modulated Array by Nonuniform Period Modulation," IEEE Antennas and Wireless Propagation Letters, vol. 14, pp. 606-609, 2015.

[8] A. Tennant and B. Chambers, "A Two-Element Time-Modulated Array With Direction-Finding Properties," Antennas Wirel. Propag. Lett., vol. 6, no. 11, pp. 64-65, 2007.

[9] C. He, X. Liang, Z. Li, J. Geng, and R. Jin, "Direction Finding by Time-Modulated Array With Harmonic Characteristic Analysis," IEEE Antennas Wirel. Propag. Lett., vol. 14, pp. 642-645, 2015.

[10] B. Clark and J. A. Flint, "Acoustical Direction Finding with TimeModulated Arrays," Sensors, vol. 16, no. 12, p. 2107, dec 2016. 